\title{
VOLTAGE GATED NANOFLUIDIC CHIP FOR PROTEIN CAPTURE, AMPLIFICATION, AND RELEASE \\ Kaushik K. Rangharajan ${ }^{1}$ and Shaurya Prakash ${ }^{1 *}$ \\ ${ }^{1}$ Department of Mechanical and Aerospace Engineering, The Ohio State University, Columbus, Ohio, USA
}

\begin{abstract}
We report on a nanofluidic 'field-effect' gated chip, with addressable gate electrodes to capture, amplify, and release a model protein, Bovine Serum Albumin (BSA). Fluidically isolated gates, embedded strategically along the length of the nanofluidic chip, when addressed facilitates generation of localized and spatially varying electric-field, driving protein amplification, via electromigration, as opposed to diffusion driven amplification observed in existing state-of-art systems. A complementing numerical model was solved to capture the changes to the local space-charge density due to gating inside the nanofluidic chip, driving pre-concentration of BSA at various segments.
\end{abstract}

\section{INTRODUCTION}

Protein trafficking through nanometer scaled channels are ubiquitous in several biological processes. While microfluidic platforms have shown potential for protein pre-concentration via high-voltage concentration polarization methods [1], nanofluidic systems provide exclusive size and charge based selectivity to sense, trap, and amplify proteins for potential applications in proteomics, bioanalysis, clinical diagnosis, gene sequencing, and drug development. Nanofluidic platform can therefore serve as a central platform to trap proteins in their native environment. Voltage gated nanofluidic devices, geometrically analogous to solid state field effect transistors, are an attractive platform to re-configurably modulate the concentration of charged proteins in vitro [2].

Previously efforts to demonstrate field-effect control of proteins were achieved inside a nanofluidic channel consisting of an embedded gate electrode patterned over the entirety of the nanochannel $[2,3]$. The net protein pre-concentration is therefore governed by the magnitude of electrostatic interaction between the charge protein and the entire charged nanofluidic wall. By modulating the polarity of the gate voltage, effective channel surface charge was altered, regulating protein transport inside the nanochannel in a diffusion limited pathway [2].

The purpose of this paper is to reduce the time-scale for protein pre-concentration by driving the amplification process via electromigration as opposed to diffusion. To do so, individually addressable gate electrodes were patterned using lithography techniques on top of an $\mathrm{SiO}_{2}$ dielectric, that fluidically isolated the metal gate from the nanochannel. The gate electrodes were located strategically along the length of the nanofluidic chip. When addressed with varying magnitudes of gate potential, localized and spatially varying electric fields (e-field) were generated inside the nanofluidic chip, driving protein amplification via electromigration.

\section{THEORY}

Ion concentration inside nanofluidic channels can be modified by altering the wall surface charge [2-9]. The potential drawback of modifying the surface chemistry of the wall via passive surface modification techniques is an irreversible, permanently altered wall surface charge state [4]. The wall surface charge can also be transiently controlled via ap plication of transverse potential gradient through a metal gate which is typically separated from the nanofluidic channel walls via a thin strip $(50-100 \mathrm{~nm})$ of dielectric $[5,6]$. With $\psi$ and $\phi$ defined as potential distribution function inside the nanochannel and dielectric respectively, and $\psi_{0}$ defined as the surface potential of the wall, the boundary condition at the surface of the dielectric-electroly te interface in the presence of a gate (along $y$, Fig. 1) is given by Equations 1 - 3 [7],

$$
\begin{gathered}
-\varepsilon_{0} \varepsilon_{d} \frac{d \phi}{d y}+\varepsilon_{0} \varepsilon_{f} \frac{d \psi}{d y}=-\sigma_{s} \\
\phi=\left(\psi_{0}-V_{g}\right) \frac{y}{\delta}+\psi_{0} \\
\frac{d^{2} \psi}{d y^{2}}=-\frac{\sum_{i} F c_{i} z_{i}}{\varepsilon_{0} \varepsilon_{f}}
\end{gathered}
$$

Where $\delta, F, \varepsilon_{0}, \varepsilon_{d}$, and $\varepsilon_{f}$ are the dielectric thickness, Faraday constant, permittivity of free space, relative permittivity of the dielectric, and electrolyte respectively. Application of positive gate potential to a negatively charged surface (Fig. 1) increases the coion (negatively charged) concentration inside the nanochannel to satisfy Equation 1. Equation 2 indicates that the potential drops linearly across the dielectric from a magnitude of $V_{g}$ at the gate to a constant surface potential of $\psi_{0}$ at the nanochannel wall. To produce a significant alteration to the potential distribution and ion concentration inside the nanochannel, $\mathrm{d} \phi / \mathrm{d} y$ must be non-trivial. This can be achieved by either increasing the applied gate potential or decreasing the dielectric thickness.
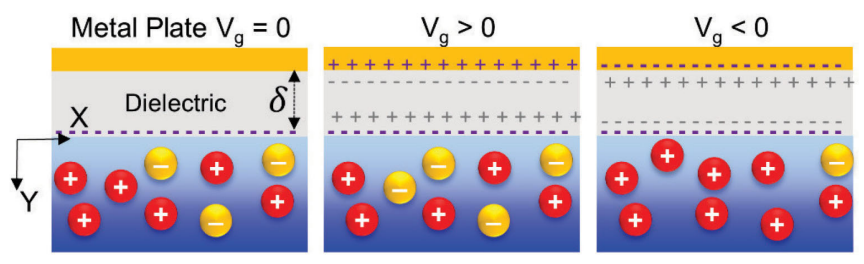

Figure 1: Schematic showing modulation of ion concentration inside nanochannel due to field-effect gating. Application of positive gate bias $\left(V_{g}>0\right)$ increases co-ion concentration and negative bias $\left(V_{g}<0\right)$ increases counter-ion concentration when compared to zero bias.

\section{METHODS}

\section{Numerical Model}

A coupled numerical model was solved in COMSOL Multiphysics (v5.2a) to calculate the electric-field and ion distribution inside the model gated nanofluidic device (Fig. 2). Poisson equation (electrostatics) was coupled with Nernst-Planck equation (Transport of diluted species) and Navier-Stokes equation (fluid flow) [8]. The surface charge density of the nanochannel and reservoir walls were assigned $-5 \mathrm{mC} / \mathrm{m}^{2}$ [9]. Specific gates were defined on top of the $40 \mathrm{~nm}$ thick dielectric. No-slip boundary conditions were defined for all walls. Reservoir protein concentration was fixed to $0.1 \mathrm{mM}$ for the simulations, matching experimental condition. A custom meshing scheme was defined and refined, until mesh insensitive solutions were obtained.

\section{Experimental Methodology}

Our proof-of-concept nanofluidic field-effect chip dramatically advances our past work on fundamental investigation of ion transport with a "single gate" from Hilton Head 2014 and 2016 [10, 

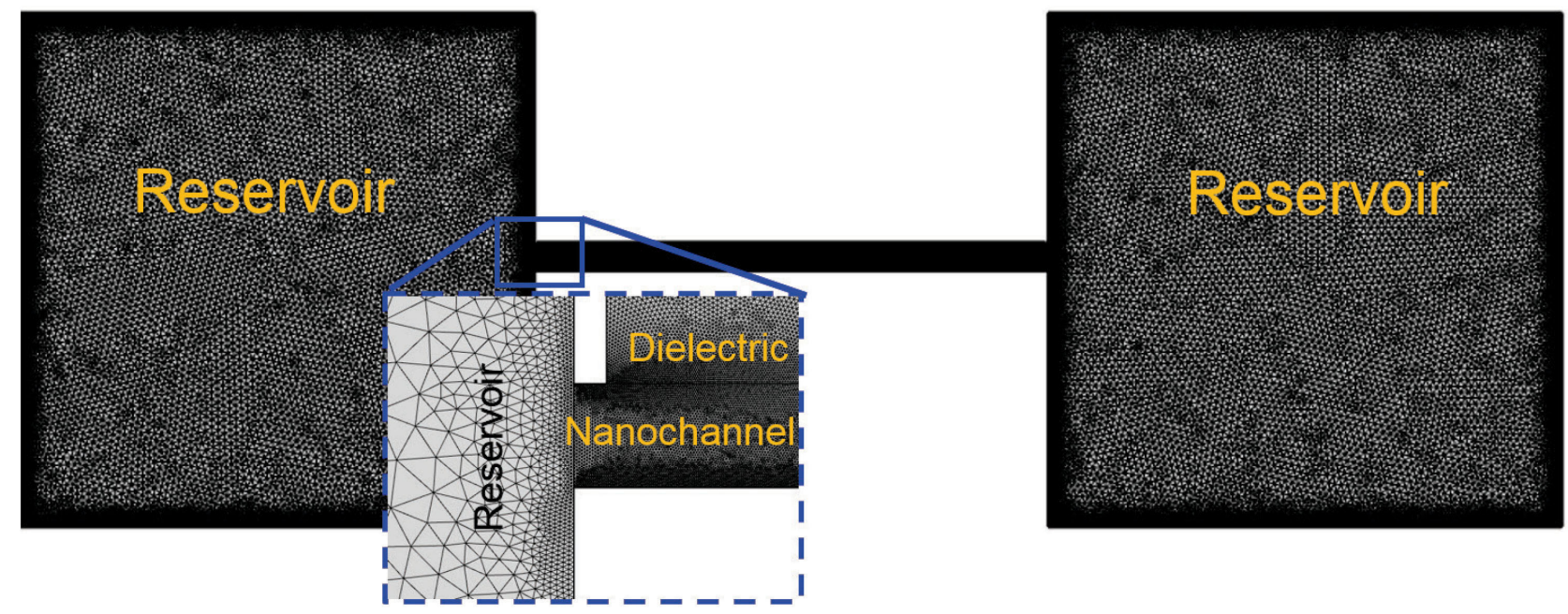

\section{Nernst-Planck Equation}

$$
\begin{aligned}
& \nabla \cdot \mathbf{J}_{i}=0 \\
& \mathbf{J}_{i}=-D_{i} \nabla c_{i}-\xi_{i} z_{i} F c_{i} \nabla \psi+c_{i} \mathbf{u}
\end{aligned}
$$

Figure 2: Geometry of the Reservoir-Nanochannel-Dielectric 'field-effect' gated system. Coupled Nernst-Planck, Poisson, and Navier-Stokes equation were solved in COMSOL Multiphysics (v5.2a) to estimate the spatially varying electric-field induced inside the nanochannel due to gating. Here, $\mathbf{J}_{\mathbf{i}}$ is the specied flux constituting the diffusion, electro-migration and convection components. For a given species $i, D_{i}$ is the diffusion coefficient, $\xi_{i}$ is the ionic mobility, $z_{i}$ is the ion valence, $\mathbf{u}$ is the convective velocity. In addition, $\rho_{s}$ is the volumetric space charge density, $\mu$ is the electrolyte viscosity and $p$, the pressure distribution inside the reservoir-nanochannel system.

11]. The device uses two microfluidic channels $(8 \mu \mathrm{m}(\mathrm{H}) \times 50 \mu \mathrm{m}$ (W) $\times 3 \mathrm{~cm}$ (L)) as fluidic reservoirs for a bank of three nanochannels $16 \mathrm{~nm}$ deep $(30 \mu \mathrm{m}(\mathrm{W})$ x $2.5 \mathrm{~mm}(\mathrm{~L}))[10,11]$. An overview of top cover fabrication with embedded individually addressable electrodes is given in Fig. 3. Each electrode is made of Chromium and is $10 \mathrm{~nm}$ high and $30 \mu \mathrm{m}$ wide.

\section{AZ 5214E Chromium}

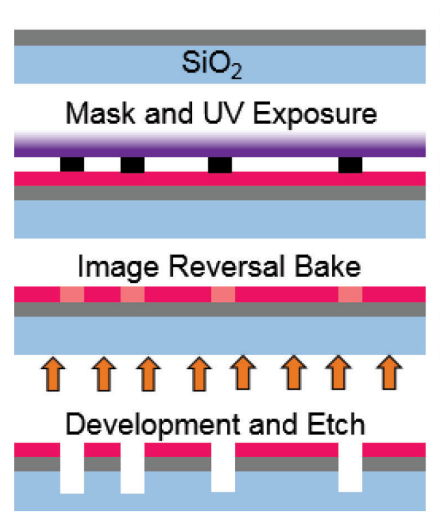

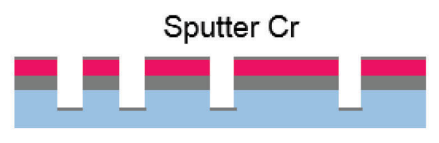

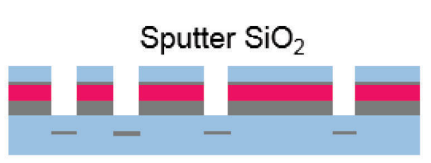

PR Lift-off / Cr Mask etch

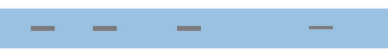

Etch gate ports and bond

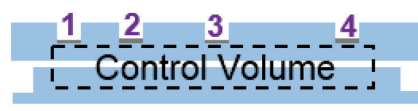

Figure 3: Fabrication sequence for embedding multiple gate electrodes on glass slide.

Several considerations were taken into account during the top cover fabrication. Chromium has a higher thermal conductivity compared to soda lime glass which helps to achieve adequate baking of photoresist AZ5214 during image reversal bake. Absence of $\mathrm{Cr}$ metal mask resulted in photoresist peeling during development. Second, a UV exposure of 3.5 secs and short development $(35 \mathrm{sec})$ was chosen to have a straight side-wall profile of AZ5214. Having a negative side-wall profile (typical during a negative PR development) causes insufficient dielectric coverage during dielectric sputtering, shorting the channel when a potential is applied. Third, $\mathrm{Cr}$ and $\mathrm{SiO}_{2}$ were sputtered at the same batch, to ensure proper insulation of $\mathrm{Cr}$. Adequate $\mathrm{SiO}_{2}$ coverage over the $\mathrm{Cr}$ electrode was verified by immersing the top cover in a Chromium etchant solution, followed by piranha cleaning for 15 minutes. Fourth, to remove inconsistencies in the deposition rate while sputtering $\mathrm{Cr}$ and $\mathrm{SiO}_{2}$, deposition rate was calibrated before each batch. The fabricated top cover electrodes and the channel slide were then bonded using the Calcium bonding recipe [12]. The final schematic of the bonded nanofluidic channel, with the multielectrode top cover is shown in Figure 4. The microfluidic and nanofluidic channels were initially filled with $0.1 \mathrm{mM}$ Bovine Serum Albumin $(66.5 \mathrm{kDa}$, stokes diameter $=6.96 \mathrm{~nm})$ conjugated with Tetramethylrhodamine (TRITC, $\lambda_{e x} / \lambda_{e m}=541 / 572 \mathrm{~nm}$ ) in Phosphate-buffered saline ( $\mathrm{pH} 7.4$ ). Potential difference of $2 \mathrm{~V}$ ( $\left.\mathrm{V}_{\text {gate }}\right)$ was applied between gates 1-2 and gates 3-4 simultaneously (Fig. 4).

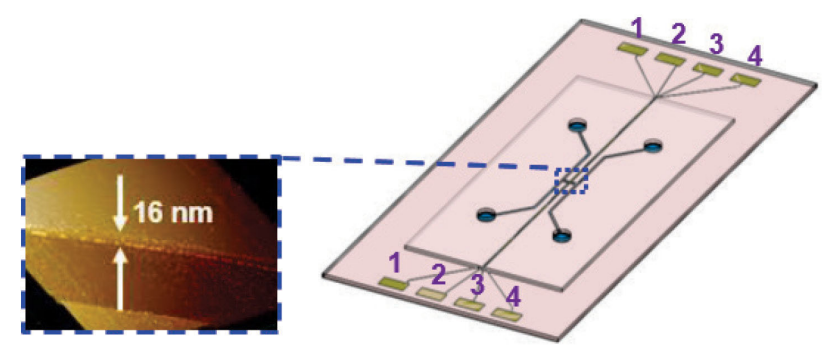

Figure 4: Schematic of bonded nanofluidic chip with inset showing AFM image of $16 \mathrm{~nm}$ dep nanochannel. 


\section{RESULTS}

Universal gate Vs Local gate

Coupled multi-physics models using COMSOL Multiphysics showed that compared to a universal gate (Fig. 5a), application of gate potential in the present system (Fig. 5 b) results in spatially varying non-uniform potential distribution in the gated and ungated region (Fig. 5c,d). In the case of a universal gate, no changes to the potential was observed inside the nanofluidic channel (Fig. 5 a, c). Application of gate potential in the present system causes a linear drop in potential across the $40 \mathrm{~nm}$ thick dielectric (Fig. 5d). To satisfy Equation 1, the local space charge density inside the nanofluidic channels is altered. Gating therefore, introduces a nonlinear gradient in potential distribution inducing a non-uniform electric field inside the nanochannel (Fig. 5c, d).
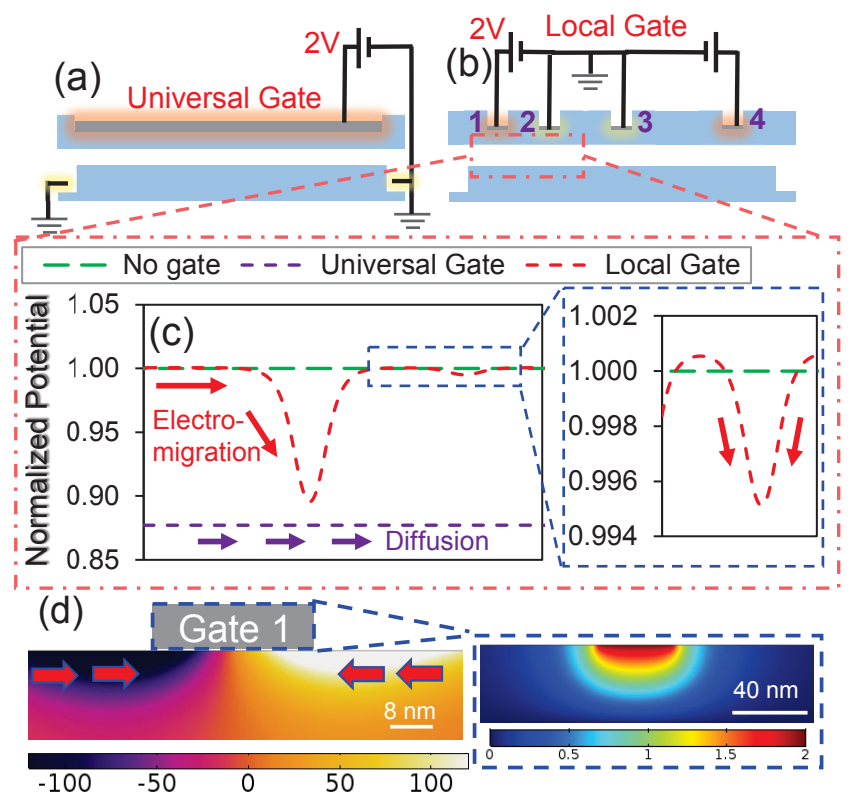

Figure 5: Design, potential distribution, and working principle of a (a) 'universally' gated nanofluidic channel and (b) individually addressable multi-electrode nanofluidic chip (present work). (c) Representative plots showing local potential changes inside the nanofluidic channels in the present device between gates 1 and 2 compared to uniform potential (similar to flow by diffusion) in the 'universally' gated nanochannel. Voltage inside the nanochannel under no gating is normalized to a value of 1 . Application of gate potential modifies volumetric space charge density inside the nanochannel to satisfy electro-neutrality, resulting in modified potential distribution compared to ungated scenario [3, 5]. (d) A non-linear (and non-uniform) electric field inside the nanochannel drives the charged proteins via electromigration for faster amplification. Electric fields of opposing polarity at the either ends of gate 1, facilitate amplification of BSA transport (Red arrows) inside the nanochannel. Inset shows potential distribution (in $\mathrm{V}$ ) inside the $40 \mathrm{~nm}$ deep $\mathrm{SiO}_{2}$ dielectric layer.

\section{BS A Capture, Amplification, and Release}

The non-uniform electric field was used to tune the preconcentration ofBSA at various segments of the nanochannel in a time-dependent manner (Fig. 6) for amplification (preconcentration) and subsequent release of BSA. The e-field facilitates BSA capture and amplification via electromigration up to $\sim 6 \mathrm{x}$ (Fig. $6 \mathrm{~b}, \mathrm{e}$ ) the initial concentration (Fig. 6a, e) in gates 2, 3 within $20 \mathrm{~min}$. The depleted BSA at gates 1, 4 are subsequently replenished by a negative e-field on the far-side of the gates (Fig. 5c) that amplifies BSA concentration by $\sim 6 \mathrm{x}, 9 \mathrm{x}$ at gates 1,4 respectively. Upon removing $\mathrm{V}_{\text {gate }}, \sim 50 \%$ of trapped BSA is "released" driven by diffusion from all the gates within next 90 min (Fig. 6d, f). In the absence of applied gate potential, amplification or release was not observed during $120 \mathrm{~min}$ of imaging.

(a)

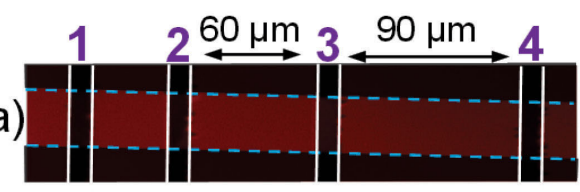

(b)

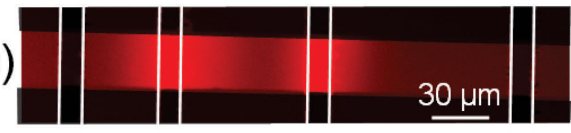

(c)

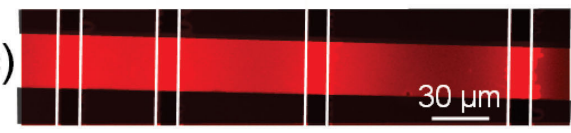

(d)

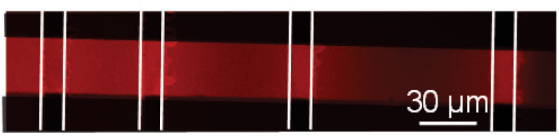

(e)
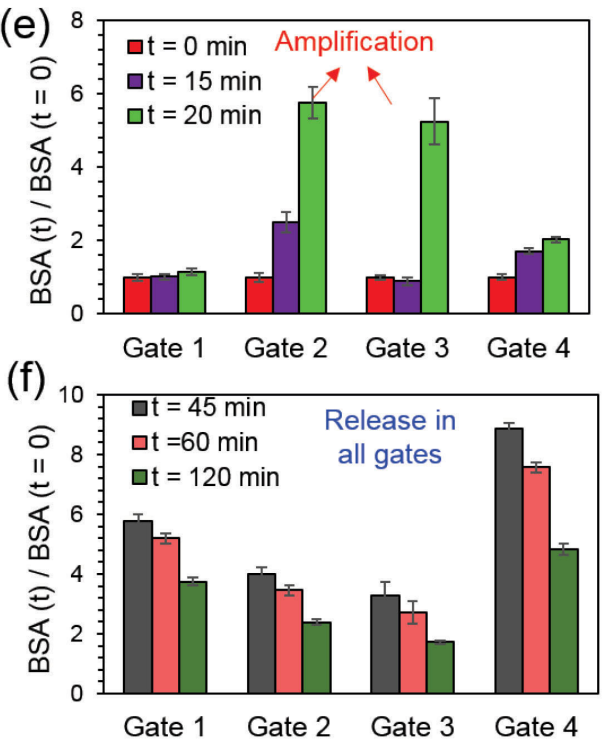

Figure 6: Fluorescence of BSA-TRITC inside the $16 \mathrm{~nm}$ deep channel was imaged when gates 1, 4 was set to a potential of $+2 \mathrm{~V}$ while gates 2, 3 were grounded (exposure time $\sim 30$ s). Fluorescence images of BSA amplification at (a) $t=0 \mathrm{~min}$, (b) $t=20 \mathrm{~min}$ and (c) $t=30 \mathrm{~min}$. (d) Representative BSA "Release" phase fluorescence image at $t=120 \mathrm{~min}$ indicating reduction in BSA-TRITC concentration. Blue dashed line indicate nanochannel boundary in Fig. $6 a$ and white-solid line indicates gated regions in Fig. $6 a-d$. (e) At $t=15 \mathrm{~min}, B S A$ concentration at gate 2 is $\sim 2.5 x$ higher than gate 3 , as the shorter distance $(30 \mu \mathrm{m})$ between gates 1 and 2, compared to $90 \mu \mathrm{m}$ between gates 3 and 4 promote a higher local field to trap BSA at gate 2. At $t=20 \mathrm{~min}, B S A$ concentration saturates to $5.5 x$ initial concentration at gates 2 and 3. (f) Switching off gate voltage at $t=30$ min leads to gradual diffusion driven protein release over the next $90 \mathrm{~min}$. 
In summary, a novel systemwas demonstrated to trap and enrich protein concentrations using electrostatic field effect gating inside a nanofluidic chip with spatially inhomogeneous electric fields providing a new avenue for protein analysis.

\section{ACKNOWLEDGMENTS}

This work was supported by The Office of Energy and Environment at The Ohio State University, US Army Research Office (ARO) through grant W911NF09C0079 and the National Science Foundation (NSF) through grant CBET-1335946. The authors acknowledge computational support and research license from the Ohio Supercomputer Center (OSC), the OSU Nanotech West cleanroom staff for assistance in fabrication.

\section{REFERENCES}

[1] J. H. Lee, S. Chung, S. J. Kim, and J. Han, "Poly(dimethylsiloxane)-Based Protein Preconcentration Using a Nanogap Generated by Junction Gap Breakdown", Analy tical Chemistry, 79, 1868 (2007).

[2] R. Karnik, K. Castelino, and A. Majumdar, "Field-effect Control of Protein Transport in a Nanofluidic Transistor Circuit", Applied Physics Letters, 88, 123114 (2006).

[3] T. James, et al., "Voltage-Gated Ion Transport through Semiconducting Conical Nanopores Formed by Metal Nanoparticle-Assisted Plasma Etching", Nano Letters, 12, 3437 (2012).

[4] E. C. Yusko, et al., "Controlling Protein Translocation through Nanopores with Bio-inspired Fluid Walls", Nature Nanotechnology, 6, 253 (2011).

[5] M. Fuest, C. Boone, K.K. Rangharajan, A.T. Conlisk, and S. Prakash, "A three-state nanofluidic field effect switch", Nano Letters, 15, 2365 (2015).

[6] R, Karnik, R. Fan, M. Yue, D. Li, P. Yang, and A. Majumdar, "Electrostatic control of ions and molecules in nanofluidic transistors"Nano Letters, 5, 943 (2005).

[7] X. Jin, and N. Aluru, "Gated transport in nanofluidic devices", Microfluidics and Nanofluidics, 11, 297 (2011).

[8] W. Guan, R. Fan, and M. Reed, "Field-effect reconfigurable nanofluidic ionic diodes", Nature Communications, 2, 506 (2011).

[9] M. Fuest, K.K. Rangharajan, C. Boone, A.T. Conlisk, and S. Prakash, "Cation dependent surface charge regulation in gated nanofluidic devices", Analytical Chemistry, 89, 1593 (2016).

[10] M. Pinti, H. A. Zambrano, K. K. Rangharajan, A.T. Conlisk, and S. Prakash, "Active Surface Potential Control for Artificial Ion Pumping", Technical Digest Solid-State Sensors, Actuators, and Microsystems Workshop, Hilton Head Island, South Carolina, June 8 - 12, (2014).

[11] C. Boone, V. Lochab, M. Fuest, and S. Prakash, "Timedependent Gating in Nanofluidic Channels for Active Electric Double Layer Modulation for Ion Transport Control", Technical Digest Solid-State Sensors, Actuators, and Microsystems Workshop, Hilton Head Island, South Carolina, June 5-9, (2016).

[12] P. B. Allen, et al., "Calcium-Assisted Glass-to-Glass Bonding for Fabrication of Glass Microfluidic Devices," Analytical Chemistry, 80, 7153 (2008).

\section{CONTACT}

*S. Prakash; Prakash.31@osu.edu 